\title{
What's the evidence for COVID-19 booster shots?
}

\author{
Cite as: CMAJ 2021 September 7;193:E1400-1 doi: 10.1503/cmaj.1095959
}

Posted on cmajnews.com on August 20, 2021.

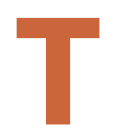

he United States government will roll out COVID-19 booster shots in late September to shore up protection against more transmissible variants. Other countries may follow suit. But the move is raising questions about the scientific and ethical basis for the decision, with some experts calling it premature.

Americans will be eligible to receive a third dose of the Pfizer or Moderna vaccines eight months after receiving their second dose. People who received the singledose Johnson \& Johnson vaccine will probably need boosters too but are not yet eligible pending further data.

The announcement came a few days after the US Food and Drug Administration (FDA) and Centers for Disease Control and Prevention (CDC) recommended COVID-19 booster shots for people with compromised immune systems. However, those agencies still need to evaluate the safety and effectiveness of a third dose for the rest of the population before the booster program starts.

\section{Why is the US moving ahead on third doses?}

Federal officials say they're launching the booster program to stay one step ahead of breakthrough infections among fully vaccinated people. They noted that COVID-19 vaccines are still highly effective, but their protection against infection appears to be waning.

"Fortunately, the vast majority of [breakthrough infections] are quite mild and what we really want to prevent is that turning into people being sick enough to be in the hospital," US National Institutes of Health Director Dr. Francis Collins told CBC Power \& Politics. "There is a hint that we might be on the path towards that, and it looks like in course of the next month or two that's going to become more of an issue."
Data from the New York State Department of Health, the Mayo Clinic and the CDC's reporting system for nursing homes showed declining protection against infection but no significant changes in severe disease or hospitalizations among fully vaccinated people.

However, as of Aug. 15, Israel reported 514 hospitalizations for severe or critical COVID-19 - up 31\% from four days earlier - and nearly three in five of those patients were fully vaccinated. That country is now offering boosters to people over 50 .

According to US President Joe Biden, "We have a responsibility to give the maximum amount of protection." He also noted that additional shots are "the best way to protect ourselves from new variants that could arise."

Some experts have noted that logistics may also factor in the decision, as it may take months to get the booster program up to full speed, even after the September start date.

\section{What's the evidence supporting the decision? from prior infection or from having more vaccinated people around them.}

White House officials presented evidence from several recent studies on infections among fully vaccinated people.

One study of residents at nearly 15000 long-term care facilities found that the protection against infection offered by the Pfizer and Moderna vaccines dropped from $74 \%$ in the spring to $53 \%$ this summer. The authors counted infections with no symptoms in their analysis and didn't look at whether vaccine effectiveness against serious illness had changed.

A New York state study that also included people who received the Johnson \& Johnson vaccine likewise reported a drop in protection against lab-confirmed infection from $92 \%$ in early May to $80 \%$ in late July. However, effectiveness against COVID-19 requiring hospitalization held steady at about $95 \%$.
Meanwhile, a preprint Mayo Clinic analysis of more than 80000 Americans found the Pfizer vaccine was only $42 \%$ effective against SARS-CoV-2 infection in July, while Moderna's was $76 \%$ effective. Even so, protection against hospitalization remained high at $75 \%$ and $81 \%$, respectively.

These findings indicate that COVID-19 vaccines are still doing an excellent job keeping fully immunized people out of hospital, even if larger numbers are testing positive for SARS-CoV-2 infection (many of whom may have no symptoms).

According to the authors of the New York state study, multiple factors may contribute to the apparent decline in vaccine effectiveness against infection from the spread of more transmissible variants to the easing of mask mandates and other public health precautions over the spring and summer. At the same time, a growing proportion of unvaccinated people may have some level of protection

\section{Where does Canada stand on vaccine boosters?}

Canada's National Advisory Committee on Immunization has not made formal recommendations on COVID-19 booster shots but is examining vaccine effectiveness in people with compromised immune systems. According to $\mathrm{NACl}$, immunocompromised people may require boosters to produce a comparable immune response to the general population.

A spokesperson for Health Canada and the Public Health Agency of Canada told CMAJ that provinces and territories may choose to offer additional doses to specific populations to enhance their protection against COVID-19. But doing so would be considered off-label use 
since Health Canada has not authorized a three-dose regimen for any of the COVID-19 vaccines.

Ontario recently announced it will offer third doses to transplant recipients and other people whose immune systems are compromised. These patients will receive boosters at least eight weeks after their second dose.

Third doses will also be available five months after immunization to residents of long-term care facilities, First Nations eldercare lodges and "higher-risk" retirement homes, although it is unclear what homes fall into this last category.

Dawn Bowdish, Canada Research Chair in Aging and Immunity, has been following vaccine effectiveness in long-term care residents, most of whom were vaccinated early this year. In a preprint study looking at residents of eight Ontario nursing homes between March and July, about one in five had no neutralizing antibodies against the virus, suggesting they may need a different vaccination regimen to achieve the same protection as the general population.

Bowdish and colleague's paper was one of several that informed Ontario's booster policy. In a complementary preprint study, Toronto researchers looked at residents in long-term care homes and found they had a much less robust immune response and fewer neutralizing antibodies than vaccinated staff.

However, Bowdish cautioned against extrapolating from older adults to the wider population because people in long-term care are "very immunologically vulnerable."

\section{Why do some experts think it's premature to offer boosters?}

According to Bowdish, the data supporting booster shots for the general population is "sparse and frankly, in Canada, it's not existent yet." She added that the
US decision seemed based on the recent reports of hospitalizations in Israel, most of which were among seniors, rather than on the local data on mostly mild and asymptomatic breakthrough infections.

Dr. Zain Chagla, an infectious disease physician at St. Joseph's Healthcare Hamilton and associate professor at McMaster University, agreed that the evidence for a general booster program is "not necessarily there yet, but it probably does need to be in the cards in the next six months to a year."

Over the next few months, Canadian jurisdictions will need to monitor breakthrough cases, particularly in vulnerable populations, he said.

South of the border, some experts argue it doesn't make sense to offer boosters while many people are still unvaccinated and COVID-19 vaccines are still highly effective.

According to Dr. Celine Gounder, an epidemiologist and infectious disease specialist at the NYU School of Medicine, improving overall vaccine uptake would offer greater community protection than giving boosters to vaccinated people.

Protection against serious disease may also last much longer than protection from infection, Gounder told NPR. "Injected vaccines like we have for COVID induce good systemic immunity in the main organs of the body," meaning that breakthrough upper-respiratory infections typically won't progress into serious illness affecting the lungs and other organs.

Memory B cells and T cells play a crucial role in fighting off severe disease but are more difficult to measure than the antibody immunity involved in preventing infection.

"The $T$ cells generated by the vaccines are holding up amazingly well, with [up to] $99 \%$ of the hospitalizations being among unvaccinated adults in the US," tweeted Dr. Monica Gandhi, an infectious disease physician at the University of California San Francisco.

Others, including a former FDA director of vaccines research, have noted that the US appears to be putting the cart before the horse by setting a September launch date when health authorities haven't evaluated the safety and effectiveness of the proposed booster program.

Pfizer only applied to the FDA to approve a third dose of its vaccine on Aug. 16 and the agency hasn't evaluated that application yet. Moderna hasn't filed an application. Meanwhile, the CDC's independent vaccine advisory committee won't start evaluating the data on boosters until next week.

Some also argue it's unethical to give boosters to prevent mild illness and asymptomatic infections in vaccinated people when many countries still have limited access to COVID-19 vaccines.

"In Canada, we have procured more vaccine doses than our population needs for every eligible adult and child over age 12 . That being the case, now is the time to send excess doses to jurisdictions around the globe where no vaccines are available," said Eleanor Fish, a professor of immunology at the University of Toronto.

\section{Lauren Vogel and Diana Duong, CMAJ}

Content licence: This is an Open Access article distributed in accordance with the terms of the Creative Commons Attribution (CC BY-NC-ND 4.0) licence, which permits use, distribution and reproduction in any medium, provided that the original publication is properly cited, the use is noncommercial (i.e., research or educational use), and no modifications or adaptations are made. See: https://creativecommons.org/ licenses/by-nc-nd/4.0/ 